\title{
Selective organ preservation with neo-adjuvant chemotherapy for the treatment of muscle invasive transitional cell carcinoma of the bladder
}

Updated online 26 May 2016: This article was originally published under a CC BY-NC-SA 4.0 license, but has now been made available under a CC BY 4.0 license. The PDF and HTML versions of the paper have been modified accordingly

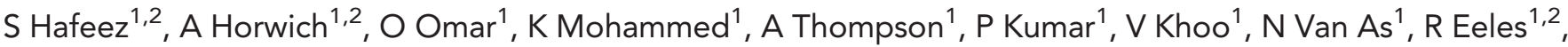 \\ D Dearnaley ${ }^{1,2}$ and R Huddart ${ }^{\star, 1,2}$ \\ ${ }^{1}$ The Royal Marsden NHS Foundation Trust, London, UK and ${ }^{2}$ The Institute of Cancer Research, London, UK
}

\begin{abstract}
Background: Radiotherapy for muscle invasive bladder cancer (MIBC) aims to offer organ preservation without oncological compromise. Neo-adjuvant chemotherapy provides survival advantage; response may guide patient selection for bladder preservation and identify those most likely to have favourable result with radiotherapy.

Methods: Ninety-four successive patients with T2-T4aNOM0 bladder cancer treated between January 2000 and June 2011 were analysed at the Royal Marsden Hospital. Patients received platinum-based chemotherapy following transurethral resection of bladder tumour; repeat cystoscopy ( \pm biopsy) was performed to guide subsequent management. Responders were treated with radiotherapy. Poor responders were recommended radical cystectomy. Progression-free survival (PFS), disease-specific survival (DSS) and overall survival (OS) were estimated using Kaplan-Meier method; univariate and multivariate analyses were performed using the Cox proportional hazard regression model.
\end{abstract}

Results: Response assessment was performed in 89 patients. Seventy-eight (88\%) demonstrated response; 53 (60\%) achieved complete response (CR); 74 responders had radiotherapy; 4 opted for cystectomy. Eleven (12\%) demonstrated poor response, 10 received cystectomy. Median survival for $\mathrm{CR}$ was 90 months (95\% Cl 64.7, 115.9) compared with 16 months (95\% $\mathrm{Cl} 5.4,27.4$; $P<0.001)$ poor responders. On multivariate analysis, only response was associated with significantly improved PFS, OS and DSS. After a median follow-up of 39 months (range 4-127 months), 14 patients (16\%) required salvage cystectomy (8 for non-muscle invasive disease, 5 for invasive recurrence, 1 for radiotherapy related toxicity). In all, $82 \%$ had an intact bladder at last follow-up after radiotherapy; $67 \%$ had an intact bladder at last follow-up or death. Our study is limited by its retrospective nature.

Conclusions: Response to neo-adjuvant chemotherapy is a favourable prognostic indicator and can be used to select patients for radiotherapy allowing bladder preservation in $>80 \%$ of the selected patients.

Radical cystectomy with pelvic node dissection remains the global standard for the treatment of muscle invasive bladder cancer (MIBC) (Gakis et al, 2013). Although advances in continent diversions, nerves sparing procedures and minimally invasive surgical techniques have been made a significant proportion of patients remain unsatisfied over postoperative

*Correspondence: Dr R Huddart; E-mail. Robert.huddart@icr.ac.uk

Received 2 November 2014; revised 12 February 2015; accepted 23 February 2015; published online 21 April 2015

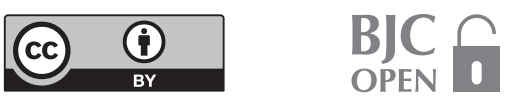


continence, sexual function and overall quality of life (Hautmann et al, 1999; Kessler et al, 2005; Pruthi et al, 2010). Therefore, in the absence of functional substitutes, it remains important to consider if it is possible to retain native bladder function while maintaining oncological outcomes. Recent bladder preservation studies have demonstrated that this can be achieved using multi-modality treatment with cure rates comparable to contemporary cystectomy series (Coppin et al, 1996; Hoskin et al, 2010; Efstathiou et al, 2012; James et al, 2012; Mak et al, 2014; Ploussard et al, 2014).

Critical to favourable outcome with bladder preservation is appropriate patient selection (Gakis et al, 2013). Selective bladder preservation protocols use a predictive marker to aid decision-making regarding definitive treatment. Complete response (CR) to induction treatment is associated with improved survival and has been suggested as a way to help identify individuals who may be particularly suitable for bladder sparing (Splinter et al, 1992; Grossman et al, 2003; Sternberg et al, 2003). Cystectomy is reserved for local recurrence as a radical salvage option with no adverse impact on subsequent survival or incidence of metastatic disease (Rödel et al, 2002). Poor response to induction treatment and low chance of cure with bladder preservation proceed directly to radical cystectomy.

The most widely investigated induction strategy employs transurethral resection of the bladder tumour (TURBT) and induction chemo-radiotherapy. Cystoscopy is performed after approximately $40 \mathrm{~Gy}$ with complete responders receiving a consolidative course of chemo-radiotherapy to a total dose of $64 \mathrm{~Gy}$. Using this technique, approximately $70 \%$ of patients will have preserved their own bladders after a median follow-up of 7.7 years (Efstathiou et al, 2012). The drawbacks are that all patients will receive some radiation and the gap during the course of radiotherapy to assess response may have adverse radiobiological implications by prolonging overall treatment time theoretically favouring tumour repopulation (Steel, 2002).

Neo-adjuvant cisplatin combination chemotherapy has an absolute survival benefit (approximately $5 \%$ at 5 years) irrespective of whether patients then proceed to cystectomy or radiotherapy and is recommended for those who are suitable (Advanced Bladder Cancer Meta-analysis Collaboration, 2003; Advanced Bladder Cancer (ABC) Meta-analysis Collaboration, 2005; Griffiths et al, 2011). Selective bladder preservation using response to neoadjuvant chemotherapy would be an alternative approach but has been less frequently investigated (Sternberg et al, 2003).

The only phase 3 study to determine the use of neo-adjuvant chemotherapy alone to guide patient selection for radical radiotherapy closed due to poor recruitment (Paramasivan et al, 2011). In the absence of randomised control studies, here we report on our 10-year experience of selective organ preservation with radiotherapy using neo-adjuvant chemotherapy.

\section{MATERIALS AND METHODS}

This analysis is of an institutional-approved protocol identifying patients treated with a selective bladder preservation approach using neo-adjuvant chemotherapy at the Royal Marsden Hospital NHS Foundation Trust between January 2000 and June 2011.

Patient eligibility. Eligible patients had histological evidence of muscle invasive transitional cell carcinoma (TCC) of the bladder and were staged according to the American Joint Committee on Cancer (Seventh edition) as T2-T4aN0M0. Radiological assessment at baseline was with computer tomography (CT) of the chest, abdomen and pelvis; magnetic resonance imaging (MRI) was the preferred imaging modality to stage the pelvis.

All patients were suitable for platinum-based neo-adjuvant chemotherapy and both radical cystectomy and radical radiotherapy.
They were required to have adequate bladder function, absence of uncorrected (unstented) hydronephrosis, adequate renal function for platinum chemotherapy, absence of widespread or distant carcinoma in situ (CIS), willingness to undergo neo-adjuvant chemotherapy with no preference for definitive treatment and no objection to long-term endoscopic follow-up if radiotherapy was recommended.

Those participating in the phase 3 randomised control study (SPARE, CRUK/07/011) were not included; their outcomes have been presented separately (Huddart et al, 2012).

Modified Charlson-Deyo score (measure of comorbidity across multiple organ sites, captured using International Classification of Diseases, Ninth revision, Clinical Modification codes) was retrospectively calculated for each patient excluding his or her diagnosis of MIBC (Deyo et al, 1992). The presence of hydronephrosis and hydroureter at presentation was also recorded, including whether ureteric stenting was performed.

Treatment. Patients were treated with initial TURBT. In accordance with clinical guidelines, safe thorough TURBT was recommended. Following TURBT, patients received 3-4 cycles of neo-adjuvant chemotherapy. This was methotrexate, vinblastine, Adriamycin (doxorubicin) and cisplatin (accelerated MVAC, also known as dose dense MVAC; 14-day cycle with granulocyte colony-stimulating factor, GCSF) or gemcitabine with cisplatin (GC); alternative platinum combination chemotherapy were also permitted (Table 1).

Assessment of response was made with repeat cystoscopy and tumour site biopsy (where possible) 3 weeks after neo-adjuvant chemotherapy. Although endoscopic assessment was preferred as the gold standard in occasional circumstances, radiological evaluation (CT and/or MRI) was also necessary in order to support clinical decision-making.

CR was defined as lack of residual tumour. If tumour site biopsy identified no residual disease, this was deemed to be a pathological $\mathrm{CR}$; if no disease was visible at endoscopy to biopsy, this was deemed to be a complete CR. Partial response (PR) was defined as either pathologically down staging to $\mathrm{pTa}, \mathrm{pT} 1, \mathrm{pT}$ is or radiological evidence of response. Poor response was defined as residual muscle invasive disease on biopsy (pT2) or radiological evidence of disease progression. Poor responders were recommended immediate radical cystectomy; responders proceeded to radical radiotherapy to the bladder (64-68 Gy in $2 \mathrm{~Gy}$ per fraction). After 2004, concurrent chemo-radiotherapy was introduced (mitomycin and 5-flurouracil, see Table 1).

Follow-up. Those patients treated with radical radiotherapy were followed up on a cystoscopic surveillance schedule. Patients with recurrence were promptly considered for either intra-vesical therapy or radical salvage cystectomy depending on the nature of the local recurrence.

Outcomes. Overall survival (OS) and progression-free survival (PFS) were defined as time from the start of chemotherapy to death from any cause and interval from chemotherapy initiation to relapse (radiological or clinical) or death, respectively. Diseasespecific survival (DSS) was defined as surviving the protocol treatment and having no evidence of distant metastases, nodal recurrence or local recurrence within the bladder that could not be salvaged with curative intent.

Surviving patients, PFS and those lost to follow-up were censored at the last assessment date. Median time to PFS, OS and DSS were estimated using Kaplan-Meier method. Univariate and multivariate analysis were performed using the Cox proportional hazard regression model. All variables with $P$-value $<0.20$ were used in the forward stepwise method for the multivariate model. Chi-squared analyses were performed to 
Table 1. Chemotherapy regimes used

\begin{tabular}{|c|c|c|c|c|c|}
\hline Neo-adjuvant chemotherapy regime & Drug & & & & \\
\hline \multirow[t]{5}{*}{ Accelerated MVAC } & & Day 1 & Day 2 & Days 4-11 & 14-Day cycle \\
\hline & Methotrexate $30 \mathrm{mg} \mathrm{m}^{-2}$ i.v. & - & & & \\
\hline & Doxorubicin $30 \mathrm{mg} \mathrm{m}^{-2}$ i.v. & & - & & \\
\hline & Cisplatin $70 \mathrm{mg} \mathrm{m}^{-2}$ i.v. & & $\bullet$ & & \\
\hline & GCSF s.c. & & & - & \\
\hline \multirow{2}{*}{ Gemcitabine-cisplatin } & Gemcitabine $1000 \mathrm{mg} \mathrm{m}^{-2}$ i.v. & - & - & & \\
\hline & Cisplatin $70 \mathrm{mg} \mathrm{m}^{-2}$ i.v. & $\bullet$ & & & \\
\hline \multirow[t]{3}{*}{ Gemcitabine-carboplatin } & & Day 1 & Day 8 & & 21-Day cycle \\
\hline & Gemcitabine $1000 \mathrm{mg} \mathrm{m}^{-2}$ i.v. & - & $\bullet$ & & \\
\hline & Carboplatin AUC 4.5 & $\bullet$ & & & \\
\hline MMC-5FU & $5 F U 500 \mathrm{mg} \mathrm{m}^{-2}$ i.v. & - & - & $\bullet$ & \\
\hline
\end{tabular}

evaluate the effect of covariates on achieving a response to neoadjuvant chemotherapy. A $P$-value $<0.05$ was considered statistically significant. All analyses were carried out using SPSS v.22 (IBM, Chicago, IL, USA).

\section{RESULTS}

Between January 2000 and June 2011, 94 successive patients with T2-T4aN0M0 bladder TCC underwent neo-adjuvant chemotherapy with the intention to be treated with a selective bladder preservation approach. Median age was 65 years (range 34-83 years). Patient characteristics are shown in Table 2.

Deviation from standard cisplatin-based neo-adjuvant chemotherapy regime occurred in 12 patients who received carboplatin and gemcitabine (5 had hydronephrosis at diagnosis; 4 had renal impairment with no hydronephrosis; 1 patient had preexisting tinnitus; in 1 patient age was cited, he was 83 years old; and in 1 patient the reason was unrecorded). Summary of chemotherapy regime response rate is presented in Table 3.

Postchemotherapy response assessment was performed in 89 patients. Two patients declined assessment expressing a preference for radiotherapy ( 1 patient) or surgery ( 1 patient); 2 patients died before their assessment, 1 from confirmed pulmonary embolism at postmortem following 2 cycles of accelerated MVAC chemotherapy, the other from non-neutropenic lower respiratory tract infection complicated by pulmonary embolism after 3 cycles of accelerated MVAC. One patient proceeded directly to radiotherapy because he had become unfit for radical surgery following an episode of neutropenic sepsis after 1 cycle of carboplatin and gemcitabine chemotherapy.

Response assessment and definitive treatment. Endoscopic assessment of response was made in 76 patients. For 13 patients, response assessment was made on radiology alone.

Seventy-eight out of $89(88 \%)$ assessable patients demonstrated a favourable response to neo-adjuvant chemotherapy. Fifty-three patients (60\%) achieved CR, including 44 (49\%) confirmed with negative tumour bed biopsy. Twenty-five patients (28\%) demonstrated PR. Eleven (12\%) patients demonstrated poor response.

Seventy-four patients who demonstrated response went on to have radical radiotherapy; 4 opted for cystectomy. All 11 patients demonstrating poor response were recommended radical cystectomy; however, 1 patient opted for radical radiotherapy. The patient study flow is presented in Figure 1.

On chi-squared analysis, there was no significant association between tumour and patient characteristics and response to neoadjuvant chemotherapy apart from the presence of hydronephrosis, where the trend demonstrated that those with hydronephrosis had poor response $(P=0.037)$.

Outcome and survival. After a median follow-up of 39 months (4-127 months), 52 (55\%) patients were alive and disease free, and 36 (38\%) had died. Median PFS, OS and DSS were 36.9 months (95\% CI 27.9, 46.0 months), 90.3 months (95\% CI 42.2, 138.4 months) and 112.4 months (95\% CI 69.5, 155.3 months), respectively. Table 4 summarises the outcome grouped by response to neo-adjuvant chemotherapy and definitive treatment.

Following radical cystectomy, 50\% (8 out of 15) experienced disease recurrence ( 2 patients with pelvic recurrences and 6 with distant metastases). Following radical radiotherapy, 47\% (36 out of 77) patients experienced disease recurrence. In all, $70 \%$ (25 out of 36) of first recurrences after radiotherapy occurred within the bladder. Also, 25\% (19 out of 77) developed non-muscle invasive bladder cancer; 10 were treated with intra-vesical therapy alone, 8 required cystectomy ( 2 for intra-vesical treatment failure; 6 as primary salvage), and one patient declined further treatment following TURBT. A total of $8 \%$ (6 out of 77) developed local invasive recurrence, 5 proceeded to salvage cystectomy but 1 patient was unfit for radical salvage. No intra-vesical recurrences occurred in those surviving $>5$ years after radiotherapy. One recurrence occurred 5 years after radiotherapy, this was metastatic in nature.

Seventeen of the 77 radiotherapy patients received concurrent chemotherapy. In all, $41 \%$ (7 out of 17) went on to experience disease recurrence. Also, 24\% (4 out of 17) of first recurrences occurred within the bladder ( 3 with non-muscle invasive bladder cancers and 1 with an invasive recurrence). Sub group univariate analysis demonstrated that concurrent chemotherapy did not reach statistical significance for PFS (HR 1.1 95\% CI 0.5, 2.3: $P=0.76$ ), OS (HR 1.0 95\% CI 0.4, 2.8: $P=0.93$ ) or DSS (HR $1.395 \%$ CI 0.4 , 4.1: $P=0.65)$.

During the follow-up period, only one patient required cystectomy because of radiotherapy-related urinary toxicity. 
Medium time to salvage cystectomy was 19.6 months (range 9.882.7 months). Bladder preservation rate was $82 \%$ (63 out of 77 ) of those undergoing radical radiotherapy and in $67 \%$ (63 out of 94 ) of the total patient population. Of the 20 patients whose follow-up has reached $\geqslant 5$ years, bladder preservation rate was $65 \%$ (11 out of 17 ) in the radiotherapy group and 55\% (11 out of 20 ) in all survivors beyond 5 years.

\section{Table 2. Patient characteristics}

\begin{tabular}{|l|c|}
\hline Age $^{\text {a }}$ & Median 65 years (range 34-83) \\
\hline Gender & 82 \\
\hline Male & 12 \\
Female &
\end{tabular}

\begin{tabular}{|l|c|}
\hline Stage of primary \\
\hline T2 & 70 \\
T3 & 18 \\
T4 & 6 \\
\hline
\end{tabular}

\section{Grade}

Intermediate (grade 2)

High (grade 3)

2

92

\begin{tabular}{l|c|} 
Presence of adjacent CIS at diagnosis \\
Yes & 23 \\
No & 31 \\
Unable to assess & 1 \\
No comment made & 35 \\
Pathology not available & 4 \\
\hline Charlson comorbidity index \\
0 & \\
1 & 65 \\
2 & 14 \\
$\geqslant 3$ & 9 \\
\hline
\end{tabular}

Hydronephrosis/hydroureter at presentation

\begin{tabular}{l|c} 
Absent & 85 \\
Present & 9 (4 received ureteric stenting) \\
\hline
\end{tabular}

\section{Chemotherapy schedule}

\begin{tabular}{|l|r}
\hline Gemcitabine-cisplatin & 43 \\
\hline Accleated
\end{tabular}

Accelerated MVAC

Gemcitabine-carboplatin

\begin{tabular}{l|c} 
Number of cycles & Median 3 cycles (range 1-5) \\
\hline 1 & 1 \\
2 & 3 \\
3 & 73 \\
4 & 16 \\
5 & 1
\end{tabular}

\begin{tabular}{|c|c|}
\hline \multicolumn{2}{|c|}{ Assessment of response } \\
\hline \multicolumn{2}{|c|}{ Cystoscopy and biopsy } \\
\hline Cystoscopy alone & 9 \\
\hline Radiology alone & 13 \\
\hline Not assessed & 5 \\
\hline \multicolumn{2}{|c|}{$\begin{array}{l}\text { Abbreviations: } \mathrm{ClS}=\text { carcinoma in situ; } \mathrm{MVAC}=\text { methotrexate, vinblastine, Adriamycin and } \\
\text { cisplatin. } \\
\mathrm{a}_{33} \text { patients were } \geqslant 70 \text { years; } 15 \text { patients were } \geqslant 75 \text { years. }\end{array}$} \\
\hline
\end{tabular}

Among patients achieving a CR after neo-adjuvant chemotherapy, $55 \%$ patients developed no further disease recurrence compared with $18 \%$ with poor response following chemotherapy. Median survival for complete responders was significantly better than poor responders, 90.3 months $(95 \%$ CI 64.7, 115.9) compared with 16.4 months (95\% CI 5.4, 27.4) $(P<0.001)$.

On univariate analysis, poor responders to neo-adjuvant chemotherapy had significantly worse outcome in terms of PFS, OS and DSS compared with those demonstrating any response (CR or PR) (Table 5). No significant difference in PFS, OS and DSS was seen between CR and PR (Figure 2).

The neo-adjuvant chemotherapy regime also significantly impacted on outcome. Those receiving gemcitabine-carboplatin had worse OS compared with gemcitabine-cisplatin (HR $3.695 \%$ CI 1.4, 9.6: $P=0.01$ ) and DSS (HR $2.895 \%$ CI 0.95, 8.29: $P=0.06$ ). No significant difference was seen between gemcitabine-cisplatin and accelerated MVAC in OS (HR 1.2 95\% CI 0.6, 2.8: $P=0.61$ ) or DSS (HR 0.6 95\% CI 0.2, 1.48: $P=0.24$ ).

On univariate analysis, the presence of hydronephrosis was associated with decreased PFS (HR 2.5 95\% CI 1.1, 5.7: $P=0.02$ ), OS (HR 4.9 95\% CI 2.0, 12.3: $P=0.001$ ) and DSS (HR 5.1 95\% CI 1.7, 15.8: $P=0.005)$.

On multivariate analysis of tumour and patient characteristics, only response to neo-adjuvant chemotherapy maintained statistical significance for PFS, OS and DSS when CR was compared with poor response and when $\mathrm{PR}$ was compared with poor response (Table 6).

\section{DISCUSSION}

Our long-term results support the use of neo-adjuvant chemotherapy response to guide patient selection for radical radiotherapy. This approach successfully allows organ preservation in approximately $70 \%$ of all patients and $>80 \%$ of patients receiving radiotherapy with survival comparable to recent surgical series (Gakis et al, 2013).

Following radiotherapy, the majority $(70 \%)$ of relapses occurred within the bladder and were amenable to subsequent salvage treatment. Encouragingly, only $6(8 \%)$ patients have developed invasive disease. Most (76\%) were non-muscle invasive recurrences, over half of which were successfully managed with TURBT and intravesical therapy, so still allowing patients to preserve functional bladders while maintaining long-term cure. Indeed after intravesical treatmen,t only 2 patients have had further relapse requiring salvage cystectomy. This pattern of relapse does raise the issue as to whether maintenance intravesical therapy should be considered in line with the management of patients with pT1G3 disease. It also means that life-long surveillance is mandated in order that prompt salvage can be implemented. These patients therefore have to be committed to regular cystoscopies, as this remains the gold standard for evaluation (Gakis et al, 2013). All intra-vesical relapses within our patient population, however, occurred within 5 years of

Table 3. Summary of chemotherapy regime response rate

\begin{tabular}{|c|c|c|c|c|c|}
\hline \multirow[b]{2}{*}{ Chemotherapy regime } & \multicolumn{5}{|c|}{ Favourable response of those assessed (\%) } \\
\hline & Total & Not assessed & Complete & Partial & $\begin{array}{l}\text { Poor response of } \\
\text { those assessed (\%) }\end{array}$ \\
\hline All & 94 & 5 & $53(60 \%)$ & $25(28 \%)$ & $11(12 \%)$ \\
\hline Gemcitabine-cisplatin & 43 & 0 & $29(67.4 \%)$ & $10(23.3 \%)$ & $4(9.3 \%)$ \\
\hline Accelerated MVAC & 39 & 3 & $20(55.6 \%)$ & $12(33.3 \%)$ & $4(11.1 \%)$ \\
\hline Gemcitabine-carboplatin & 12 & 2 & $4(40 \%)$ & $3(30 \%)$ & $3(25 \%)$ \\
\hline
\end{tabular}




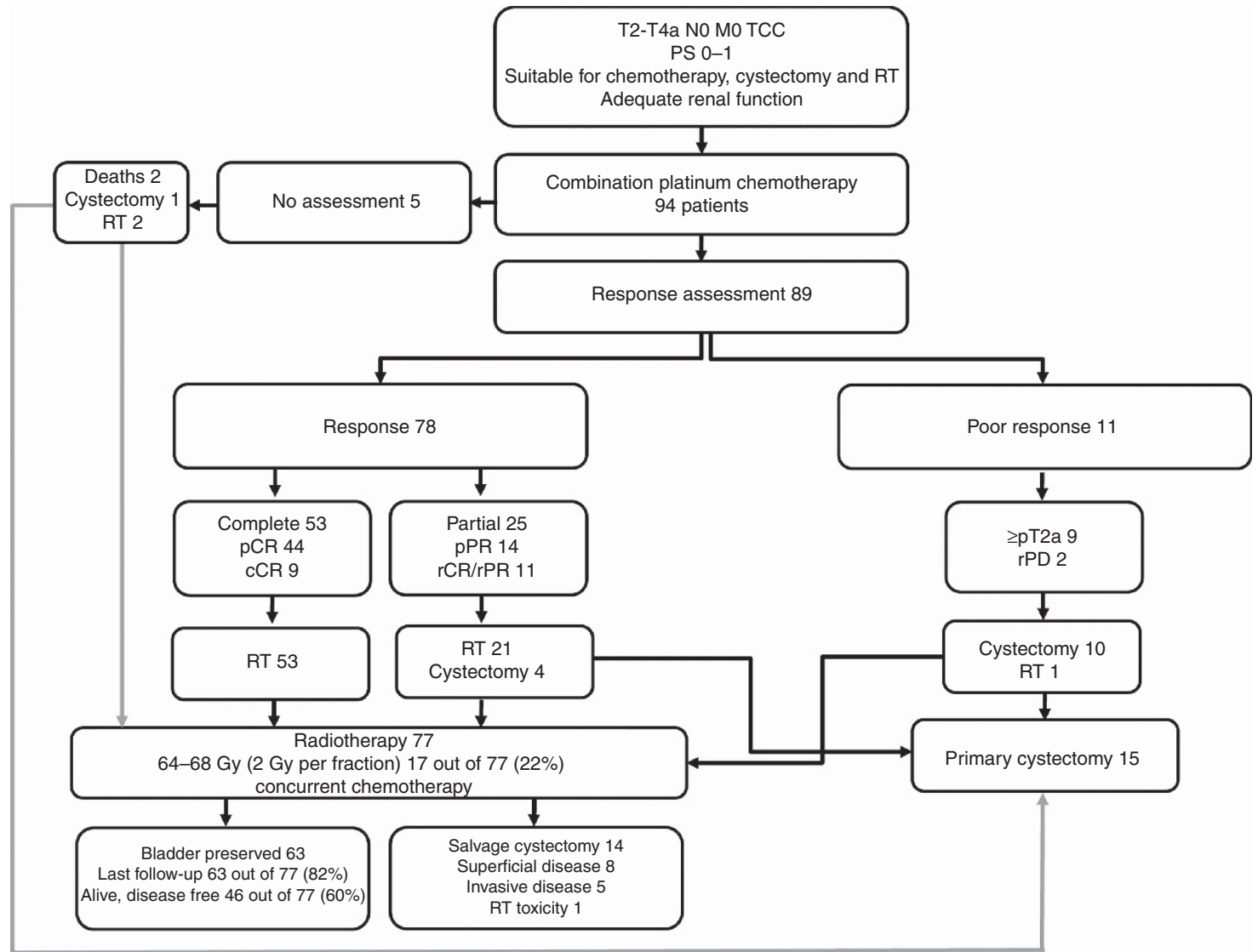

Figure 1. Patient study flow. $c C R=$ clinical complete response; $P C R=$ pathological complete response; $P D=$ disease progression; $\mathrm{PPR}=$ pathological partial response; $\mathrm{PS}=$ performance status; $\mathrm{rCR}=$ radiological complete response; $r \mathrm{PR}=$ radiological partial response; $\mathrm{RT}=$ radiotherapy

\section{Table 4. Status at last follow-up}

\begin{tabular}{|c|c|c|c|c|c|}
\hline & \multicolumn{3}{|c|}{ Treatment response to neo-adjuvant chemotherapy } & \multicolumn{2}{|c|}{ Definitive treatment $^{a}$} \\
\hline & Complete & Partial & Poor & Radiotherapy & Cystectomy \\
\hline Alive & $37 / 53$ & $17 / 25$ & $2 / 11$ & $52 / 77$ & $6 / 15$ \\
\hline Disease free & 31 & 17 & 2 & 46 & 6 \\
\hline Localised disease (bladder) & 4 & - & - & 4 & - \\
\hline Local regional disease (pelvis) & 1 & - & - & 1 & - \\
\hline Metastases & 1 & - & - & 1 & - \\
\hline Dead & $16 / 53$ & $8 / 25$ & $9 / 11$ & $25 / 77$ & $9 / 15$ \\
\hline Metastases & 10 & 4 & 8 & 15 & 8 \\
\hline Other malignancy & 4 & 1 & - & 5 & - \\
\hline Other causes & 2 & 1 & 1 & 3 & 1 \\
\hline Unknown & - & 2 & - & 2 & - \\
\hline
\end{tabular}

completing radiotherapy; further evidence and longer follow-up may help to inform the actual intensity of cystoscopic surveillance beyond 5 years.

It has been suggested that the presence of CIS on original biopsy should be a contraindication to bladder preservation. However, on the limited data we have available we find no evidence to support this assertion.

Although no formal assessment of bladder function or quality of life was performed, only one patient required cystectomy for radiotherapy-related urinary toxicity. Previous work corroborates acceptable genito-urinary and gastro-intestinal toxicity following radiotherapy and chemo-radiotherapy (Zietman et al, 2003; Efstathiou et al, 2009; Efstathiou et al, 2012; James et al, 2012).

The historical nature of the cohort meant that the majority of patients received radiotherapy alone; only $22 \%$ received concurrent chemo-radiotherapy. Phase 3 evidence supports he use of mitomycin and 5-flurouracil to improve local-regional diseasefree survival compared with radiotherapy alone (HR $0.6895 \% \mathrm{CI}$, 0.48 to $0.96 ; P=0.03$ ) (James et al, 2012). Thus the universal use of these or other radiosensitising agents could further improve the local control rates reported here (Hoskin et al, 2010; Choudhury et al, 2011; Gakis et al, 2013; Mitin et al, 2013). 


\section{Table 5. Univariate analyses for survival end points}

Variable

Events $(n=55)$

Median time, months $(95 \% \mathrm{Cl})$

$\mathrm{HR}(95 \% \mathrm{Cl})$

$P$-value

\section{Progression-free survival}

\begin{tabular}{|c|}
\hline Age: \\
$\leqslant 65$ \\
$>65$ \\
\hline Gender \\
Female \\
Male \\
\hline T stage \\
T2 \\
T3 \\
T4
\end{tabular}

Chemotherapy regime

Gemcitabine-cisplatin

Gemcitabine-carboplatin Acc-MVAC

Response

Complete

Partial

Poor

Number of cycles

2-3

4-5

Presence of CIS at diagnosis ${ }^{\mathrm{b}}$

CIS

No CIS

Hydronephrosis

No

Yes

Charlson score

0

1

$\geqslant 2$

3
3
24

\begin{tabular}{l|l}
31 & $37.2(25.0-49.9)$ \\
24 & $36.2(13.6-58.7)$
\end{tabular}

$5 \quad$ Not estimable ${ }^{a}$

36.9 (24.4-49.5)

$39 \quad 39.4(27.1-51.7)$

4

Variable

$\mid$

$(1$

\begin{tabular}{c|c}
1 & 0.81 \\
$1.07(0.62-1.84)$ & \\
\hline 1 & 0.37 \\
$1.53(0.60-3.85)$ & \\
\hline
\end{tabular}

0.81

Overall survival

\begin{tabular}{|l}
\hline Age \\
$\leqslant 65$ \\
$>65$ \\
\hline Gender \\
Female \\
Male \\
\hline T stage \\
T2 \\
T3 \\
T4
\end{tabular}

Chemotherapy regime

Gemcitabine-cisplatin

Gemcitabine-carboplatin Acc-MVAC

Response

Complete

Partial

Poor

Number of cycles

2-3

4-5

Presence of CIS at diagnosis ${ }^{b}$

CIS

No CIS

Hydronephrosis

No

Yes

Charlson score

0

1

$\geqslant 2$

$34.6(13.2-56.0)$

$14.1(0-36.1)$

12
4

(2)

$23 \quad 37.2(25.6-48.8)$

$19.6(12.4-26.8)$ $40.5(30.2-50.8)$

$40.5(34.9-46.2)$

$40.0(14.6-65.4)$

$14.0(6.5-21.6)$

$39.4(33.5-45.2)$

$25.1(15.9-34.3)$

$39.4(5.3-73.5)$

$30.8(13.1-48.4)$

$39.4(32.7-46.0)$

$17.3(11.9-22.7)$

$36.9(19.3-54.6)$

$39.4(30.5-48.3)$

$40.0(16.1-63.9)$

Events $(n=36)$

Median time, months $(95 \% \mathrm{Cl})$

1

$1.28(0.67-2.45)$

$1.64(0.58-4.61)$

1

$1.38(0.59-3.23)$

$0.84(0.47-1.51)$

1

$1.14(0.60-2.16)$

$3.84(1.78-8.25)$

$1.36(0.69-2.66)$

$1.25(0.61-2.54)$

1

$2.54(1.13-5.70)$

1

$0.60(0.25-1.41)$

$1.20(0.55-2.58)$

HR $(95 \% \mathrm{Cl})$

\begin{tabular}{l|l}
19 & $102.7(31.5-173.8)$
\end{tabular}

\begin{tabular}{l|r}
17 & $76.0(33.0-119.0)$
\end{tabular}

Not estimable ${ }^{a}$

90.3 (34.0-146.6)

2
34

$9.3(34.0-146.6)$

$102.7(51.9-153.5)$

$40.5(13.0-68.1)$

$44.7(0-100.0)$

Not estimable ${ }^{a}$

32.1 (14.6-49.7)

$90.3(59.1-121.5)$

90.3 (64.7-115.9)

Not estimable ${ }^{a}$

$16.4(5.41-27.4)$

$1.16(0.5-2.71)$

1

$1.51(0.76-3.00)$

$2.58(0.62-10.82)$

1

$2.21(1.04-4.68)$

$1.976(0.581-6.715)$

1

$3.61(1.37-9.55)$

$1.23(0.55-2.77)$

1

8.94 (3.77-21.19)

90.3 (42.2-138.4)

$41.8(29.6-53.9)$

1

$1.28(0.51-3.10)$

Not estimable ${ }^{a}$

Not estimable ${ }^{a}$

90.3 (62.2-118.4)

$29.6(5.7-53.6)$

90.3 (28.6-152.0)

Not estimable ${ }^{a}$

47.1 (25.3-69.0)
0.53

0.51

.37

002

0.69

0.001

0.38

0.54

0.02

.41

-value 


\begin{tabular}{|c|c|c|c|c|}
\hline Variable & Events $(n=25)$ & Median time, months ( $95 \% \mathrm{Cl})$ & $\mathrm{HR}(95 \% \mathrm{Cl})$ & $P$-value \\
\hline \multicolumn{5}{|l|}{ Disease-specific survival } \\
\hline $\begin{array}{l}\text { Age } \\
\leqslant 65 \\
>65\end{array}$ & $\begin{array}{c}16 \\
9\end{array}$ & $\begin{array}{l}112.4 \text { (73.1-151.6) } \\
76.0 \text { (not estimable }{ }^{a} \text { ) }\end{array}$ & $0.85(0.40-2.14)$ & 0.92 \\
\hline $\begin{array}{l}\text { Gender } \\
\text { Female } \\
\text { Male }\end{array}$ & $\begin{array}{c}2 \\
23\end{array}$ & $\begin{array}{l}\text { Not estimable }{ }^{a} \\
112.4(74.6-150.1)\end{array}$ & $\begin{array}{c}1 \\
1.78(0.42-7.60)\end{array}$ & 0.44 \\
\hline $\begin{array}{l}\text { T stage } \\
\text { T2 } \\
\text { T3 } \\
\text { T4 }\end{array}$ & $\begin{array}{l}16 \\
6 \\
3\end{array}$ & $\begin{array}{l}112.4(50.9-173.9) \\
\text { Not estimable } \\
\left.90.3 \text { (not estimable }{ }^{a}\right)\end{array}$ & $\begin{array}{c}1 \\
1.94(0.75-5.01) \\
2.58(0.74-8.98)\end{array}$ & 0.19 \\
\hline $\begin{array}{l}\text { Chemotherapy regime } \\
\text { Gemcitabine-cisplatin } \\
\text { Gemcitabine-carboplatin } \\
\text { Acc-MVAC }\end{array}$ & $\begin{array}{c}10 \\
5 \\
10\end{array}$ & $\begin{array}{l}\text { Not estimable } \\
\left.32.1 \text { (not estimable }{ }^{a}\right) \\
112.4(73.2-151.6)\end{array}$ & $\begin{array}{c}1 \\
2.80(0.95-8.29) \\
0.55(0.21-1.48)\end{array}$ & $\begin{array}{l}0.028 \\
0.06 \\
0.24\end{array}$ \\
\hline $\begin{array}{l}\text { Response } \\
\text { Complete } \\
\text { Partial } \\
\text { Poor }\end{array}$ & $\begin{array}{l}12 \\
4 \\
8\end{array}$ & $\begin{array}{c}112.4(79.1-145.7) \\
\text { Not estimable } \\
16.4(5.41-27.4)\end{array}$ & $\begin{array}{c}1 \\
0.79(0.25-2.44) \\
11.76(4.43-31.23)\end{array}$ & $\begin{aligned}< & 0.001 \\
& 0.68 \\
< & 0.001\end{aligned}$ \\
\hline $\begin{array}{l}\text { Number of cycles } \\
2-3 \\
4-5\end{array}$ & $\begin{array}{c}20 \\
5\end{array}$ & $\begin{array}{r}112.4(73.4-151.5) \\
\text { Not estimable }^{a}\end{array}$ & $\begin{array}{c}1 \\
1.59(0.58-4.39)\end{array}$ & 0.37 \\
\hline $\begin{array}{l}\text { Presence of CIS at diagnosis }{ }^{b}, n=17 \\
\text { CIS } \\
\text { No CIS }\end{array}$ & $\begin{array}{l}8 \\
9\end{array}$ & $\begin{array}{c}90.3 \text { (27.5-153.1) } \\
112.4 \text { (not estimable }{ }^{a} \text { ) }\end{array}$ & $\begin{array}{c}1 \\
0.77(0.28-2.14)\end{array}$ & 0.62 \\
\hline $\begin{array}{l}\text { Hydronephrosis } \\
\text { No } \\
\text { Yes }\end{array}$ & $\begin{array}{c}21 \\
4\end{array}$ & $\begin{array}{c}112.4(73.4-151.4) \\
24.6(3.5-45.7)\end{array}$ & $\begin{array}{c}1 \\
5.11(1.65-15.81)\end{array}$ & 0.005 \\
\hline $\begin{array}{l}\text { Charlson score } \\
0 \\
1 \\
\geqslant 2\end{array}$ & $\begin{array}{l}19 \\
2 \\
4\end{array}$ & $\begin{array}{r}112.4(76.6-148.2) \\
\text { Not estimable } \\
47.1(9.0-85.3) \\
\end{array}$ & $\begin{array}{c}1 \\
0.42(0.10-1.82) \\
1.47(0.49-4.44)\end{array}$ & 0.36 \\
\hline $\begin{array}{l}\text { Abbreviations: Acc-MVAC = methotrexate, vin } \\
\mathrm{CIS}=\text { carcinoma in situ; } \mathrm{HR}=\text { hazard ratio. } \\
{ }^{\mathbf{a}} \text { Not possible to estimate, as cumulative survi } \\
\mathbf{b}_{\text {Missing information regarding the presence }}\end{array}$ & $\begin{array}{l}\text { he, doxorubicin and } \mathrm{c} \\
\text { rve does not fall belon } \\
\text { ence of CIS. }\end{array}$ & $\begin{array}{l}\text { in given over } 2 \text { weeks with GCSF support } \\
\text { and the groups' survival curve did not rea }\end{array}$ & $\begin{array}{l}\text { cyte colony-stimulating } \\
\text { n. }\end{array}$ & onfidence it \\
\hline
\end{tabular}

Sixty-seven percent of local recurrences after radiotherapy occur at the original bladder tumour site, supporting the likelihood of persistent occult disease (Zietman et al, 2001). The radiation doseresponse relationship of these tumours means that higher radiation doses targeted to the bladder tumour may offer further opportunity to improve local control (Huddart et al, 2014). Partial cystectomy as an alternative organ-sparing approach does not offer universal substitution for radiotherapy as only 5\% of MIBC meet the stringent criteria necessary to ensure acceptable local control rates, including bladder dome lesions where a minimum $2-\mathrm{cm}$ margin can be removed without compromise to continence or bladder capacity (Gakis et al, 2013).

The rate of pelvic nodal relapse following radiotherapy is low, consistent with other reported series (James et al, 2012; Mak et al, 2014). Historically, bladder radiotherapy used large margins to capture geometric uncertainty of filling and motion during treatment and is likely therefore to have encompassed at-risk pelvic nodal groups to some extent, potentially delivering enough dose to sterilise disease. The recent implementation of more accurate radiotherapy (image guided and intensity modulated) has lent itself to margin reduction as certainty in dose delivered to target has improved; in turn, this could impact on incidental pelvic nodal irradiation and so future nodal relapse rates will need to be monitored. In practice, however, the question of whether pelvic irradiation in bladder cancer should be used at all remains unclear and a point of on-going debate.

Response to neo-adjuvant chemotherapy was identified as an important prognostic indicator and was the only significant predictor of survival on multivariate analysis of this cohort.
No difference in outcome was seen between those demonstrating CR or PR to chemotherapy.

Poor response to neo-adjuvant chemotherapy was associated with significantly worse PFS and OS despite radical treatment with cystectomy. The majority of poor responding patients $(73 \%, 8$ out of 11) subsequently died from metastatic disease. This is consistent with earlier series reflecting less favourable outcome for poor responders to induction therapy, with a reported 5-year DFS of $20 \%$ and $>40 \%$ of patients unfortunately developing metastases within 2 years (Rödel et al, 2002). Intensification of systemic treatment to reduce metastatic relapse should therefore be an important consideration. The molecular characterisation of MIBC opens the door to potential personalised systemic therapy with targeted agents for those unlikely to respond to conventional platinum therapy (Cancer Genome Atlas Research Network, 2014; Choi et al, 2014). The poor outcome at present, however, raises the question about the standard conventional approach to offer these patients cystectomy given that systemic disease control currently remains an issue for the majority of poor responders.

Current selective bladder preservation protocols are reliant on response to induction therapy to inform definitive treatment. This approach has a number of limitations. Chemotherapy is not without significant toxicity and potential mortality. Those who do not respond are exposed to morbidity with no certain benefit and a delay in effective treatment. The challenge remains how best to identify these patients and their tumour characteristics at diagnosis to determine individual benefit from chemotherapy and likelihood of cure with radical radiotherapy.

Many groups have performed single-marker studies but no biomarker is used routinely in the clinic to select patients 


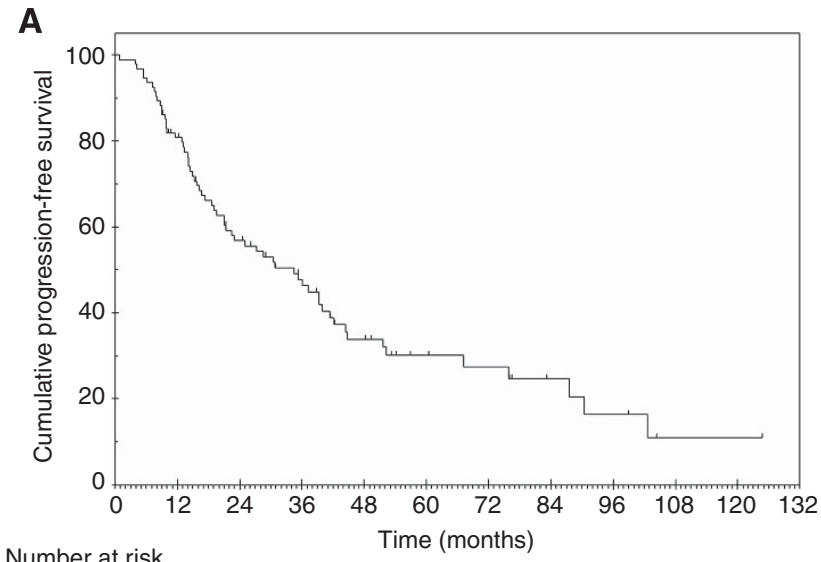

Number at risk $\begin{array}{lllllllllll}94 & 73 & 48 & 34 & 20 & 12 & 10 & 6 & 4 & 1 & 1\end{array}$

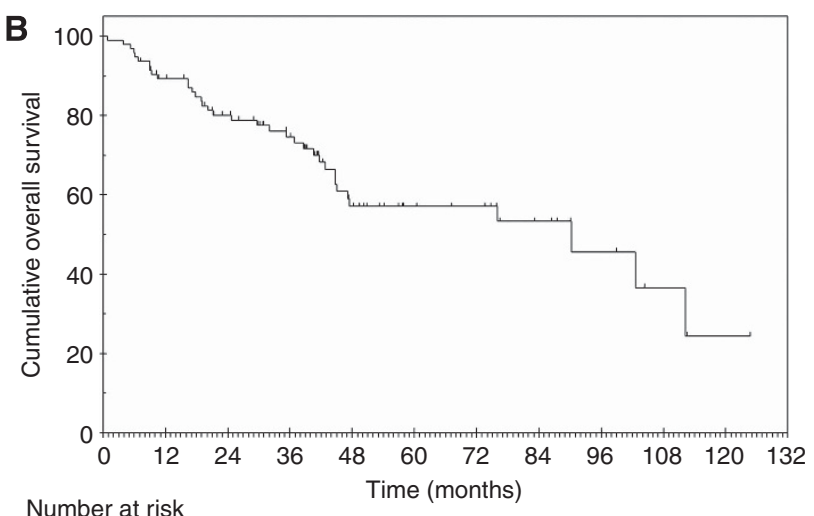

\section{C}

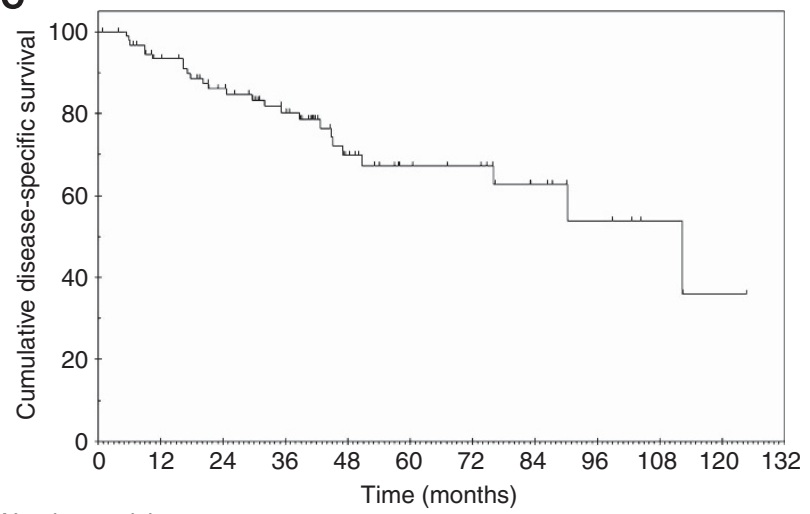

Number at risk

$\begin{array}{lllllllllll}94 & 80 & 66 & 51 & 30 & 20 & 18 & 10 & 6 & 3 & 1\end{array}$

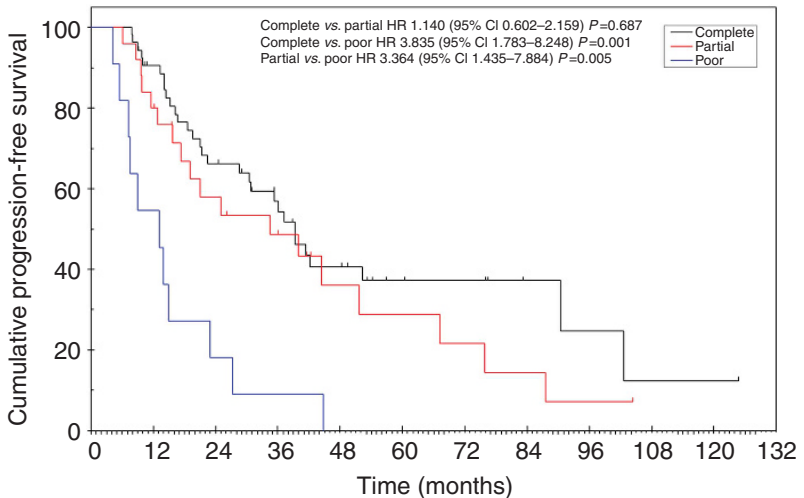

Number at risk

$\begin{array}{llllllllllll}\text { Complete } & 53 & 45 & 32 & 22 & 14 & 7 & 6 & 3 & 2 & 1 & 1\end{array}$ $\begin{array}{llllllllll}\text { Partial } & 25 & 20 & 13 & 10 & 5 & 4 & 3 & 2 & 1\end{array}$ Poor $\quad \begin{array}{llll}11 & 6 & 2 & 1\end{array}$

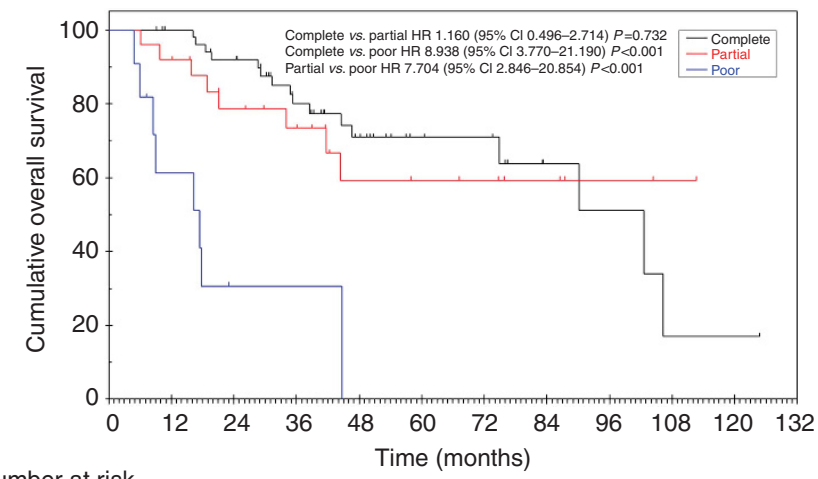

$\begin{array}{lllllllllllll}\text { Complete } & 53 & 50 & 45 & 31 & 21 & 12 & 11 & 5 & 3 & 1 & 1\end{array}$ $\begin{array}{lllllllllll}\text { Partial } & 25 & 23 & 17 & 14 & 8 & 7 & 6 & 4 & 2 & 1\end{array}$ Poor $\quad \begin{array}{llll}11 & 6 & 2 & 2\end{array}$

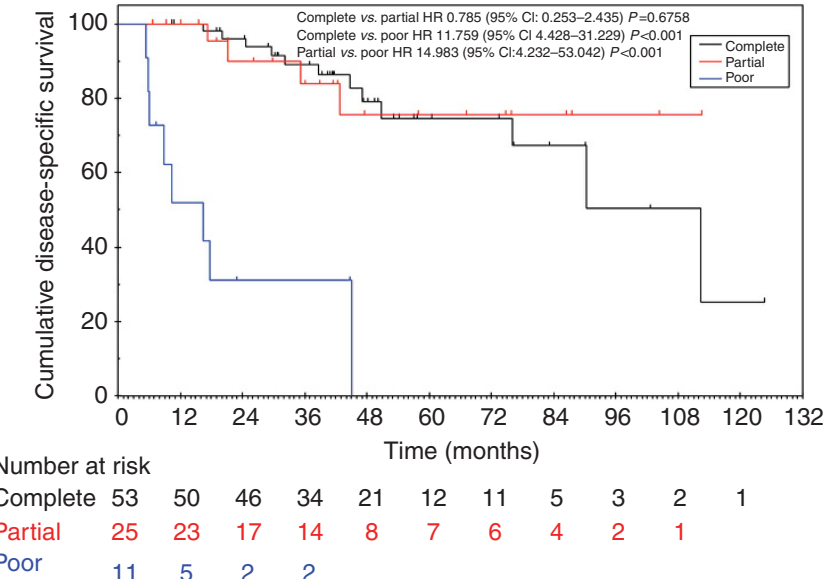

Figure 2. Kaplan-Meier plots for rates of (A) progression-free survival, (B) overall survival and (C) disease-specific survival for all patients and stratified by response to neo-adjuvant chemotherapy.

for treatment. For example, high MRE11 expression specifically predicts improved outcome with radiotherapy, demonstrating $16 \%$ improvement in 3-year cancer-specific survival (CSS) compared with high MRE11 expression treated with cystectomy (69.9\% vs 53.8\% 3-year CSS, $P=0.021$ ) (Choudhury et al, 2010). It is envisaged that MRE11 expression could be used to guide future consultations with patients about bladder radiotherapy, but prospective studies and standardised laboratory testing are needed before this transition to clinical practice can occur.
Previous studies have identified T stage as a strong predictor of outcome (Efstathiou et al, 2012). Our multivariate analysis did not reflect this possibly because during the 10 -year assessment period routine local staging changed from CT to MRI assessment and additionally relatively small numbers of patients had advanced stage disease. The majority (approximately 75\%) of patients were staged as T2 disease at presentation. Although this is consistent with other bladder radiotherapy cohorts, the known discordance between clinical and pathological staging is likely to have further confounded stratification by stage (Efstathiou et al, 2012; James 
Table 6. Multivariate analyses for survival end points

\begin{tabular}{|c|c|c|c|c|c|c|}
\hline \multirow[b]{2}{*}{ Variable $^{a}$} & \multicolumn{2}{|c|}{ Progression-free survival } & \multicolumn{2}{|c|}{ Overall survival } & \multicolumn{2}{|c|}{ Disease-specific survival } \\
\hline & $\mathrm{HR}(95 \% \mathrm{Cl})$ & $P$-value & HR $(95 \% \mathrm{Cl})$ & $P$-value & $\mathrm{HR}(95 \% \mathrm{Cl})$ & $P$-value \\
\hline Response & & 0.002 & & $<0.001$ & & 0.001 \\
\hline Complete & 1 & & 1 & & 1 & \\
\hline Partial & $1.14(0.60-2.16)$ & 0.687 & $1.16(0.50-2.71)$ & 0.732 & $0.79(0.25-2.44)$ & 0.675 \\
\hline Poor & $3.84(1.78-8.25)$ & 0.001 & 8.938 (3.77-21.19) & $<0.001$ & $11.76(4.43-31.23)$ & $<0.001$ \\
\hline
\end{tabular}

et al, 2012; Mak et al, 2014). The presence of hydronephrosis was associated with worse PFS, OS and DSS on univariate analysis and may have been proxy of advanced local disease. Hydronephrosis was associated with poor response; again suggestive of high T stage and larger tumours, but these patients were also more likely to receive carboplatin chemotherapy (56\% compared with $8 \%$ with no hydronephrosis).

Completeness of TURBT has also been reported as an important predictor of outcome, but this was difficult to verify retrospectively in our cohort (Rödel et al, 2002; Efstathiou et al, 2012). Incomplete TURBT reflects the presence of larger tumours and is a surrogate measure of advanced $\mathrm{T}$ stage.

Although the numbers are small, those who received carboplatin had a poorer OS. Though this is consistent with the evidence that carboplatin is inferior to cisplatin-containing chemotherapy regimes in both the neo-adjuvant and metastatic setting, it could be due to confounding factors leading to the decision to use carboplatin-based treatment (Advanced Bladder Cancer Metaanalysis Collaboration, 2003; Advanced Bladder Cancer (ABC) Meta-analysis Collaboration, 2005; Gakis et al, 2013).

As pathological CR can be achieved with chemotherapy and TURBT, the question whether further treatment is necessary for these patients arises. The phase II SWOG study (S0219) demonstrated no difference in survival between complete responders who went on to have cystectomy or those who had close endoscopic follow-up (70\% vs $76 \%$ respectively). Adopting a strict surveillance protocol after $\mathrm{CR}$ with radical treatment offered promptly at progression did not adversely affect OS (deVere White et al, 2009). Larger randomised studies would be necessary before those achieving a good response or CR to induction could have conventional radical treatment confidently deferred because their cancer cure had been achieved with induction alone.

\section{CONCLUSION}

Response to neo-adjuvant chemotherapy is a favourable prognostic indicator and can be used to select patients for radiotherapy allowing bladder preservation in approximately $70 \%$ of patients with survival comparable to recent surgical series.

\section{ACKNOWLEDGEMENTS}

We acknowledge NHS funding to the NIHR Biomedical Research Centre for Cancer and to Cancer Research UK (CRUK).

\section{REFERENCES}

Advanced Bladder Cancer (ABC) Meta-analysis Collaboration (2005)

Neoadjuvant chemotherapy in invasive bladder cancer: update of a systematic review and meta-analysis of individual patient data advanced bladder cancer (ABC) meta-analysis collaboration. Eur Urol 48: 202-205discussion 205-6.
Advanced Bladder Cancer Meta-analysis Collaboration (2003) Neoadjuvant chemotherapy in invasive bladder cancer: a systematic review and metaanalysis. Lancet 361: 1927-1934.

Cancer Genome Atlas Research Network (2014) Comprehensive molecular characterization of urothelial bladder carcinoma. Nature 507: 315-322.

Choi W, Porten S, Kim S, Willis D, Plimack ER, Hoffman-Censits J, Roth B, Cheng T, Tran M, Lee IL, Melquist J, Bondaruk J, Majewski T, Zhang S, Pretzsch S, Baggerly K, Siefker-Radtke A, Czerniak B, Dinney CP, McConkey DJ (2014) Identification of distinct basal and luminal subtypes of muscle-invasive bladder cancer with different sensitivities to frontline chemotherapy. Cancer Cell 25: 152-165.

Choudhury A, Nelson LD, Teo MT, Chilka S, Bhattarai S, Johnston CF, Elliott F, Lowery J, Taylor CF, Churchman M, Bentley J, Knowles MA, Harnden P, Bristow RG, Bishop DT, Kiltie AE (2010) MRE11 expression is predictive of cause-specific survival following radical radiotherapy for muscle-invasive bladder cancer. Cancer Res 70: 7017-7026.

Choudhury A, Swindell R, Logue JP, Elliott PA, Livsey JE, Wise M, Symonds P, Wylie JP, Ramani V, Sangar V, Lyons J, Bottomley I, McCaul D, Clarke NW, Kiltie AE, Cowan RA (2011) Phase II study of conformal hypofractionated radiotherapy with concurrent gemcitabine in muscle-invasive bladder cancer. J Clin Oncol 29: 733-738.

Coppin CM, Gospodarowicz MK, James K, Tannock IF, Zee B, Carson J, Pater J, Sullivan LD (1996) Improved local control of invasive bladder cancer by concurrent cisplatin and preoperative or definitive radiation. The National Cancer Institute of Canada Clinical Trials Group. J Clin Oncol 14: 2901-2907.

deVere White RW, Lara Jr PN, Goldman B, Tangen CM, Smith DC, Wood Jr DP, Hussain MH, Crawford ED (2009) A sequential treatment approach to myoinvasive urothelial cancer: a phase II Southwest Oncology Group trial (S0219). J Urol 181: 2476-2480discussion 2480-2481.

Deyo RA, Cherkin DC, Ciol MA (1992) Adapting a clinical comorbidity index for use with ICD-9-CM administrative databases. J Clin Epidemiol 45: 613-619.

Efstathiou JA, Bae K, Shipley WU, Kaufman DS, Hagan MP, Heney NM, Sandler HM (2009) Late pelvic toxicity after bladder-sparing therapy in patients with invasive bladder cancer: RTOG 89-03, 95-06, 97-06, 99-06. J Clin Oncol 27: 4055-4061.

Efstathiou JA, Spiegel DY, Shipley WU, Heney NM, Kaufman DS, Niemierko A, Coen JJ, Skowronski RY, Paly JJ, McGovern FJ, Zietman AL (2012) Long-term outcomes of selective bladder preservation by combinedmodality therapy for invasive bladder cancer: the MGH experience. Eur Urol 61: 705-711.

Gakis G, Efstathiou J, Lerner SP, Cookson MS, Keegan KA, Guru KA, Shipley WU, Heidenreich A, Schoenberg MP, Sagaloswky AI, Soloway MS, Stenzl A. International Consultation on Urologic Disease-European Association of Urology Consultation on Bladder Cancer 2012 (2013) ICUD-EAU International Consultation on Bladder Cancer 2012: radical cystectomy and bladder preservation for muscle-invasive urothelial carcinoma of the bladder. Eur Urol 63: 45-57.

Griffiths G, Hall R, Sylvester R, Raghavan D, Parmar MK (2011) International phase III trial assessing neoadjuvant cisplatin, methotrexate, and vinblastine chemotherapy for muscle-invasive bladder cancer: long-term results of the BA06 30894 trial. J Clin Oncol 29: 2171-2177.

Grossman HB, Natale RB, Tangen CM, Speights VO, Vogelzang NJ, Trump DL, deVere White RW, Sarosdy MF, Wood Jr DP, Raghavan D, Crawford ED (2003) Neoadjuvant chemotherapy plus cystectomy compared with cystectomy alone for locally advanced bladder cancer. N Engl J Med 349: 859-866. 
Hautmann RE, de Petriconi R, Gottfried HW, Kleinschmidt K, Mattes R, Paiss T (1999) The ileal neobladder: complications and functional results in 363 patients after 11 years of followup. J Urol 161: 422-427discussion 427-428.

Hoskin PJ, Rojas AM, Bentzen SM, Saunders MI (2010) Radiotherapy with concurrent carbogen and nicotinamide in bladder carcinoma. J Clin Oncol 28: 4912-4918.

Huddart R, Birtle A, Lewis R, Bahl A, Falconer A, Maynard L, Hall E (2012) Results of the SPARE feasibility study-selective bladder preservation against radical excision in muscle invasive T2/T3 transitional cell carcinoma of the bladder (CRUK/07/011). Int J Radiat Oncol Biol Phys 84: S119-S120.

Huddart R, McDonald F, Hafeez S, Warren-Oseni K, Taylor H, Thompson A, Khoo V, Harris V, McNair H, Mohammed K, Thomas K, Jones K, Dearnaley D, Horwich A (2014) Phase I dose-escalated image-guided adaptive bladder radiotherapy study: Results of first dose cohort (68Gy). J Clin Oncol 32(4_suppl): Abstract 291.

James ND, Hussain SA, Hall E, Jenkins P, Tremlett J, Rawlings C, Crundwell M, Sizer B, Sreenivasan T, Hendron C, Lewis R, Waters R, Huddart RA. BC2001 Investigators (2012) Radiotherapy with or without chemotherapy in muscle-invasive bladder cancer. N Engl J Med 366: 1477-1488.

Kessler TM, Burkhard FC, Studer UE (2005) Clinical indications and outcomes with nerve-sparing cystectomy in patients with bladder cancer. Urol Clin North Am 32: 165-175.

Mak RH, Hunt D, Shipley WU, Efstathiou JA, Tester WJ, Hagan MP, Kaufman DS, Heney NM, Zietman AL (2014) Long-term outcomes in patients with muscle-invasive bladder cancer after selective bladderpreserving combined-modality therapy: a pooled analysis of Radiation Therapy Oncology Group Protocols 8802, 8903, 9506, 9706, 9906, and 0233. J Clin Oncol 32: 3801-3809.

Mitin T, Hunt D, Shipley WU, Kaufman DS, Uzzo R, Wu CL, Buyyounouski MK, Sandler H, Zietman AL (2013) Transurethral surgery and twice-daily radiation plus paclitaxel-cisplatin or fluorouracil-cisplatin with selective bladder preservation and adjuvant chemotherapy for patients with muscle invasive bladder cancer (RTOG 0233): a randomised multicentre phase 2 trial. Lancet Oncol 14: 863-872.

Paramasivan S, Huddart R, Hall E, Lewis R, Birtle A, Donovan JL (2011) Key issues in recruitment to randomised controlled trials with very different interventions: a qualitative investigation of recruitment to the SPARE trial (CRUK/07/011). Trials 12: 78.

Ploussard G, Daneshmand S, Efstathiou JA, Herr HW, James ND, Rödel CM, Shariat SF, Shipley WU, Sternberg CN, Thalmann GN, Kassouf W (2014) Critical analysis of bladder sparing with trimodal therapy in muscleinvasive bladder cancer: a systematic review. Eur Urol 66: 120-137.

Pruthi RS, Nix J, McRackan D, Hickerson A, Nielsen ME, Raynor M, Wallen EM (2010) Robotic-assisted laparoscopic intracorporeal urinary diversion. Eur Urol 57: 1013-1021.

Rödel C, Grabenbauer GG, Kühn R, Papadopoulos T, Dunst J, Meyer M, Schrott KM, Sauer R (2002) Combined-modality treatment and selective organ preservation in invasive bladder cancer: long-term results. J Clin Oncol 20: 3061-3071.

Splinter TA, Scher HI, Denis L, Bukowski R, Simon S, Klimberg I, Soloway M, Vogelzang NJ, van Tinteren H, Herr H (1992) The prognostic value of the pathological response to combination chemotherapy before cystectomy in patients with invasive bladder cancer. European Organization for Research on Treatment of Cancer-Genitourinary Group. J Urol 147: 606-608.

Steel G (2002) Basic Clinical Radiobiology. 3rd edn (Oxford University Press. Sternberg CN, Pansadoro V, Calabrò F, Schnetzer S, Giannarelli D, Emiliozzi P, De Paula F, Scarpone P, De Carli P, Pizzo M, Platania A, Amini M (2003) Can patient selection for bladder preservation be based on response to chemotherapy? Cancer 97: 1644-1652.

Zietman AL, Grocela J, Zehr E, Kaufman DS, Young RH, Althausen AF, Heney NM, Shipley WU. (2001) Selective bladder conservation using transurethral resection, chemotherapy, and radiation: management and consequences of $\mathrm{Ta}, \mathrm{T} 1$, and Tis recurrence within the retained bladder. Urology 58: 380-385.

Zietman AL, Sacco D, Skowronski U, Gomery P, Kaufman DS, Clark JA, Talcott JA, Shipley WU (2003) Organ conservation in invasive bladder cancer by transurethral resection, chemotherapy and radiation: results of a urodynamic and quality of life study on long-term survivors. J Urol 170: $1772-1776$.

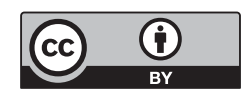

This work is licensed under the Creative Commons Attribution 4.0 International License. To view a copy of this license, visit http://creativecommons.org/licenses/by/4.0/ 